Research Article

\title{
Synchronous Vibration Parameters Identification of Variable Rotating Speed Blades Based on New Improved Two-Parameter Method without OPR Sensor
}

\author{
Liang Zhang ${ }^{\mathbb{D}},{ }^{1}$ Huiqun Yuan, ${ }^{2}$ and $\mathrm{Xin} \mathrm{Li}^{1}$ \\ ${ }^{1}$ Faculty of Mechanical Engineering and Automation, Liaoning University of Technology, Jinzhou, China \\ ${ }^{2}$ School of Mechanical Engineering and Automation, Northeastern University, Shenyang, China \\ Correspondence should be addressed to Liang Zhang; zhangliang545238@163.com
}

Received 13 April 2021; Revised 6 June 2021; Accepted 16 July 2021; Published 26 July 2021

Academic Editor: Paola Forte

Copyright (c) 2021 Liang Zhang et al. This is an open access article distributed under the Creative Commons Attribution License, which permits unrestricted use, distribution, and reproduction in any medium, provided the original work is properly cited.

Blade tip-timing is one of the most effective methods for blade vibration parameters identification of turbomachinery. Once-per revolution (OPR) sensor is usually used to determine the rotating speed and as a time reference. However, the OPR sensor leads to a large measurement error or even failure, or it is difficult to install. A new improved two-parameter method without the OPR sensor is proposed to identify the synchronous vibration parameters of variable rotating speed blades. Three eddy current sensors are required to identify the excitation order, vibration amplitude, resonance rotating speed frequency, resonance frequency, and the initial phase of the blades. Numerical simulation of blade synchronous vibration parameters identification is presented, and the identification error of the method is investigated. The simulation results show that the identification accuracy of this method is better than that of the traditional two-parameter method and the improved method in reference, especially in the identification of the vibration initial phase. Experiments are conducted based on the blade tip-timing vibration measurement system. The results indicate that the standard errors of vibration parameter identification results between the new method and the method in reference are smaller, except for the initial phase. It is consistent with the results of the simulation identification. The synchronous vibration parameter identification of variable rotating speed blades without the OPR sensor is achieved based on the new improved two-parameter method.

\section{Introduction}

Blades are crucial component of aero-engine used in a wide range of turbomachinery, including engine, compressor, and turbine $[1,2]$. The reliable operation of blades is an important factor to ensure the safety of aero-engine. $60 \%$ of aero-engine failure can be attributed to vibration, of which $70 \%$ of these failures are blade vibration failure [3]. Accurate measurement of blade vibration parameters (such as frequency, amplitude, and phase) is an important way for monitoring the safe operation of aero-engine. Therefore, there is an urgent need for online monitoring of blade vibration to ensure the safe and reliable operation of aeroengine.

In recent years, various blade vibration monitoring systems are developed. Nowadays, noncontact blade tip- timing (BTT) measurement technology, with the advantage of noncontact measuring all the blades, has obtained widespread applications in industries blade vibration monitoring $[4,5]$. BTT technology measures the vibration amplitude and frequency of the blades of a rotating disk with sensors mounted on the rig case to detect the arriving time of individual blades. The basic principle of this technique is that, in the absence of vibrations, the blade passes in front of the sensor after each rotation in a time that depends only on the rotational speed. On the contrary, in the case in which the blade is vibrating, the vibration changes the time of arrival (TOA) and the new value can be higher or lower than the theoretical one. Vibration amplitudes are extracted by subtracting arriving time to theoretical ones.

A novel BTT analysis method for reconstructing unknown multimode blade vibration signals was proposed. 
Experiments were carried out to validate the feasibility of the proposed method [6]. A new measurement configuration for a BTT system usable with optical probes, the so called beam shutter configuration, was tested by comparing the BTT results with the strain gauges results [7]. A novel method of parameter identification has been developed for blade asynchronous vibration under variable speed excitations based on the two sensors data interpolation in order to increase the frequency that can be identified [8]. An enhanced estimation of signal parameters via rotational invariance technique was proposed. The main advantage of this technique is its ability to analyze both single and multimode blade vibrations spreading across a wide dynamic range, while accommodating the effect of varying rotational speeds and sensor installation errors [9]. A noncontact and thus low interference experimental set-up for exciting and measuring blade vibrations was proposed and shown to work. A novel acoustic system excites rotor blade vibrations, which are measured with an optical tiptiming system [10]. The tip-timing method is used based on the fiber Bragg grating magnetically coupling sensor to study and analyze the ventilator blade vibration in order to realize long-distance and noncontact real-time online safety monitoring of blade vibration [11]. A new positioning for the blade tip-timing optical sensors was tested in the case of a shrouded bladed disk. A particular placement of optical laser sensors is studied in order to point at the leading and trailing edges' locations where the blades experience the largest vibration amplitudes with the aim of not interfering with the flow path [12]. A modification of an existing test rig to measure out-of-plane vibrations of a rotating bladed disc using a blade tip-timing (BTT) system and a standard SLDV system is proposed [13]. It presents a method to extract the main parameters (amplitude and frequency) in resonance condition from the tip-timing measurements. The proposed method was then applied to the BTT measured signals coming from a rotating bladed disk excited at different engine orders [14]. A method for using the capacitance probe sensor to measure both tip timing and tip clearance is proposed by correlating capacitance probe tip-timing results with simultaneously measured blade-mounted strain gauge vibration results and precise rotational speeds. Blade tip vibration amplitudes were measured using capacitance probes and compared with strain-derived vibration levels [15].

All the methods mentioned above require an OPR sensor, which can measure rotating speed, provide time reference, and locate blade tip-timing data [16, 17]. When the axial distance between the OPR sensor and the bladed disk is long, the blade tip-timing result may contain large measurement noise [18]. In some cases, the OPR sensor is difficult to install, especially on the high pressure rotors of the dual rotor engines [19]. Therefore, a blade tip-timing method without the OPR sensor is urgently required to identify the blade vibration parameters.

Without the OPR sensor, a method of obtaining OPR signal based on fitting blade tip-timing data was proposed by Russhard [20]. Chen et al. [21] proposed an efficient and high-precision compound reference based on the BTT method without OPR. The method was based on straight line fitting in reference [20], and the factors such as the blade installation angle error, probe installation angle error, and blade disk mistuning were considered. A synchronous resonance vibration measurement method of variable speed rotating blade based on tip-timing was presented without the OPR sensor [22]. This method was developed on the basis of the traditional single-parameter method, and the method utilizes tip-timing intervals of neighboring blades and the installation errors of blades to realize the identification and requires no OPR signal. In the absence of the OPR sensor, the traditional two-parameter method was improved by mounting an additional BTT sensor and the amplitude and engine order of blade vibration can be identified through analyzing the vibration displacement change [23]. The improved method is an approximate method, which leads to a certain error between the theoretical value and the identification simulation value without introducing the error.

In this paper, a new improved two-parameter method based on three eddy current blade tip-timing sensors which requires no OPR sensor is presented to identify the synchronous vibration parameters of variable rotating speed blades. Compared with the method in reference [23], this method is an accurate method, which can not lead to error between the theoretical value and the identification simulation value without introducing error.

The left outline of this paper is summarized as follows. The mathematical model of parameters identification with the new improved two-parameter method is introduced in Section 2. Theoretic error of this method is discussed in Section 3. In Section 4, experiments are conducted and the synchronous vibration parameters of the rotor blades in variable rotating speed are identified based on the experimental data. Finally, some major conclusions are summarized in Section 5.

\section{Vibration Parameters Identification Method}

2.1. Traditional Two-Parameter Method. Traditional twoparameter method, also known as two blade tip-timing sensors method, is based on the variable speed sweep frequency. An OPR sensor TIP and two blade tip-timing sensors TIP0 and TIP1 installed adjacent to each other on the casing are used to obtain the blade synchronous vibration parameters, as shown in Figure 1. The angle between TIP sensor and TIP0 sensor is 0 , and the angle between TIP0 sensor and TIP1 sensor is $\alpha$.

The single-frequency vibration displacement response of the blade can be written as follows:

$$
y(t)=A_{0}|H(\omega)| \cos \left(\omega t-\phi(\omega)+\phi_{0}\right)
$$

Here, $A_{0}$ is the displacement generated by the excitation force, $\omega$ is the vibration frequency of the blade, and $\phi_{0}$ is the initial phase.

Amplitude-frequency response is as follows:

$$
H(\omega)=\frac{1}{\sqrt{\left[1-\left(\omega / \omega_{n}\right)^{2}\right]^{2}+\left(2 \xi\left(\omega / \omega_{n}\right)\right)^{2}}} .
$$




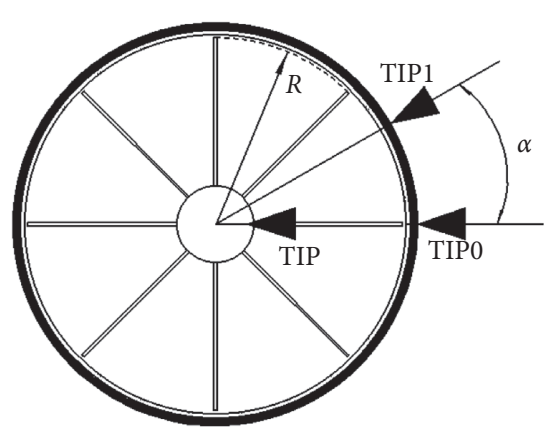

(a)

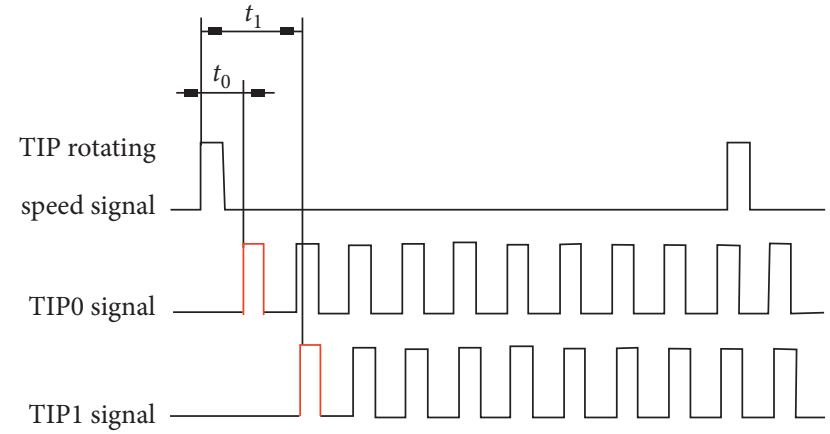

(b)

FIgURE 1: Schematic diagram of sensor layout and blade tip-timing signal of the traditional two-parameter method.

Phase-frequency response is as follows:

$$
\phi(\omega)=\arctan \frac{2 \xi \omega / \omega_{n}}{1-\left(\omega / \omega_{n}\right)^{2}} .
$$

Here, $\omega_{n}$ is the resonance angular frequency of the blade and $\xi$ is the damping coefficient. For synchronous vibration, the vibration angular frequency of the blade $\omega=N_{e} \Omega, N_{e}$ is the excitation order, and $\Omega$ is the angular frequency of the rotating speed.

The blade passes the sensor TIP0 at time $t_{0}$ and passes sensor TIP1 at time $t_{1}$. The vibration displacement of the blade passing the sensor TIP0 is $y_{0}$ and the blade passing the sensor TIP1 is $y_{1}$. According to equation (1) and considering the existence of the same vibration constant deviation $C$ in the actual, the vibration displacement of the same blade obtained by the two sensors is as follows:

$$
\left\{\begin{array}{l}
y_{0}=A_{0}|H(\omega)| \cos \left(\omega t_{0}-\phi(\omega)+\phi_{0}\right)+C \\
y_{1}=A_{0}|H(\omega)| \cos \left(\omega t_{1}-\phi(\omega)+\phi_{0}\right)+C
\end{array}\right.
$$

The origin of $y_{0}$ and $y_{1}$ and the role of vibrational constant deviation in the fitting process are described in reference [16] of the traditional two-parameter method. The two-parameter method also measures the synchronous vibration of the blade, $\omega=N_{e} \Omega$; let $t_{0}=0$, then $t_{1}=\alpha / \Omega$, and substituting it into equation (4) gives

$$
\left\{\begin{array}{l}
y_{0}=A_{0}|H(\omega)| \cos \left(-\phi(\omega)+\phi_{0}\right)+C \\
y_{1}=A_{0}|H(\omega)| \cos \left(-\phi(\omega)+N_{e} \alpha+\phi_{0}\right)+C
\end{array}\right.
$$

It can be seen from equations (2) and (3) that the amplitude-frequency response can be written as follows:

$$
|H(\omega)|=\frac{\sin (\phi(\omega))}{2 \xi\left(\omega / \omega_{n}\right)} .
$$

If the damping coefficient $\xi$ is very small, the vibration amplitude takes the maximum $A_{\max }$ when the excitation frequency is equal to the natural frequency:

$$
A_{\max }=\frac{A_{0}}{2 \xi} \text {. }
$$

Substituting equation (6) into equation (5), combined with equation (7), and after integration and difference transformation, equation (5) can be written as follows:

$$
\left\{\begin{array}{l}
y_{0}=\frac{A_{\max }}{2} \frac{\omega_{n}}{\omega}\left[\sin \left(2 \phi(\omega)-\phi_{0}\right)+\sin \left(\phi_{0}\right)\right]+C, \\
y_{1}=\frac{A_{\max }}{2} \frac{\omega_{n}}{\omega}\left[\sin \left(2 \phi(\omega)-N_{e} \alpha-\phi_{0}\right)+\sin \left(N_{e} \alpha+\phi_{0}\right)+C\right] .
\end{array}\right.
$$

The traditional two-parameter method is simplified when the up-speed sweep passes near the blade resonance region; let $\omega \approx \omega_{n}$, equation (8) is simplified as follows:

$$
\left\{\begin{array}{l}
y_{0} \approx \frac{A_{\max }}{2} \sin \left(2 \phi(\omega)-\phi_{0}\right)+\frac{A_{\max }}{2} \cdot \sin \left(\phi_{0}\right)+C, \\
y_{1} \approx \frac{A_{\max }}{2} \sin \left(2 \phi(\omega)-N_{e} \alpha-\phi_{0}\right)+\frac{A_{\max }}{2} \cdot \sin \left(N_{e} \alpha+\phi_{0}\right)+C .
\end{array}\right.
$$

Equation (9) is similar to an elliptic equation but does not fully satisfy the elliptic equation. The shortcoming of the traditional two-parameter method is that the vibration parameter identification has some theoretical errors. The fitting parameters are the center coordinates $(x 0, y 0)$ of the ellipse and the long and short axes of the standard ellipse $a$ and $b$. The detailed fitting process can be seen in reference [24] of the traditional two-parameter method. When the elliptic equation is directly fitted, there is an error in the identified vibration parameters, especially the initial phase $\phi_{0}$ with a large error.

2.2. New Improved Two-Parameter Method. The traditional two-parameter method uses an OPR sensor TIP and two blade tip-timing sensors TIP0 and TIP1. In practical applications, the OPR sensor should be installed near the rotating shaft, and the installation is complicated or there is no installation location. Based on the above shortcomings, according to the traditional two-parameter method, a new 
improved two-parameter method is proposed. The new improved two-parameter method uses three-blade tiptiming sensors TIP0, TIP1, and TIP2 instead of the OPR sensor TIP. The angle between the sensors TIP0 and TIP1; TIP0 and TIP2 are $\alpha_{1}$ and $\alpha_{2}$ as shown in Figure 2, respectively.

The blade passes the sensor TIP0 at time $t_{0}$, passes sensor TIP1 at time $t_{1}$, and passes sensor TIP2 at time $t_{2}$. The vibration displacement of the blade passing the sensor TIP0 is $y_{0}$, passing the sensor TIP1 is $y_{1}$, and the passing the sensor TIP1 is $y_{2}$. According to equation (1) and considering the existence of the vibration constant deviation $C$ in the actual, the vibration displacement of the same blade obtained by the three sensors is as follows:

$$
\left\{\begin{array}{l}
y_{0}=A_{0}|H(\omega)| \cos \left(\omega t_{0}-\phi(\omega)+\phi_{0}\right)+C, \\
y_{1}=A_{0}|H(\omega)| \cos \left(\omega t_{1}-\phi(\omega)+\phi_{0}\right)+C, \\
y_{2}=A_{0}|H(\omega)| \cos \left(\omega t_{2}-\phi(\omega)+\phi_{0}\right)+C .
\end{array}\right.
$$

The two-parameter method also measures the synchronous vibration of the blade, $\omega=N_{e} \Omega$; let $t_{0}=0$, then $t_{1}=\alpha_{1} / \Omega$ and $t_{2}=\alpha_{2} / \Omega$, and substituting it into equation (10) gives

$$
\left\{\begin{array}{l}
y_{0}=A_{0}|H(\omega)| \cos \left(-\phi(\omega)+\phi_{0}\right)+C, \\
y_{1}=A_{0}|H(\omega)| \cos \left(-\phi(\omega)+N_{e} \alpha_{1}+\phi_{0}\right)+C \\
y_{2}=A_{0}|H(\omega)| \cos \left(-\phi(\omega)+N_{e} \alpha_{2}+\phi_{0}\right)+C .
\end{array}\right.
$$

Substituting equation (6) into equation (11), combined with equation (7), and after integration and difference transformation, equation (11) can be written as follows:

$$
\begin{aligned}
& y_{0}=\frac{A_{\max }}{2} \frac{\omega_{n}}{\omega}\left[\sin \left(2 \phi(\omega)-\phi_{0}\right)+\sin \left(\phi_{0}\right)\right]+C, \\
& y_{1}=\frac{A_{\max }}{2} \frac{\omega_{n}}{\omega}\left[\sin \left(2 \phi(\omega)-N_{e} \alpha_{1}-\phi_{0}\right)+\sin \left(N_{e} \alpha_{1}+\phi_{0}\right)\right]+C, \\
& y_{2}=\frac{A_{\max }}{2} \frac{\omega_{n}}{\omega}\left[\sin \left(2 \phi(\omega)-N_{e} \alpha_{2}-\phi_{0}\right)+\sin \left(N_{e} \alpha_{2}+\phi_{0}\right)\right]+C .
\end{aligned}
$$

Equation (13) minus equation (12) and equation (14) minus equation (13) and then the following equation is obtained through the trigonometric function induction formula transformation:

$$
\begin{aligned}
\frac{\omega}{\omega_{n}}\left(y_{1}-y_{0}\right)= & A_{\max } \sin \left(\frac{N_{e} \alpha_{1}}{2}\right) \sin \left[\frac{3 \pi}{2}-\left(\frac{4 \phi(\omega)-N_{e} \alpha_{1}-2 \phi_{0}}{2}\right)\right]+\frac{A_{\max }}{2}\left[\sin \left(N_{e} \alpha_{1}+\phi_{0}\right)-\sin \left(\phi_{0}\right)\right] \\
\frac{\omega}{\omega_{n}}\left(y_{2}-y_{1}\right)= & A_{\max } \sin \left(\frac{N_{e}\left(\alpha_{2}-\alpha_{1}\right)}{2}\right) \sin \left[\frac{3 \pi}{2}-\left(\frac{4 \phi(\omega)-N_{e}\left(\alpha_{1}+\alpha_{2}\right)-2 \phi_{0}}{2}\right)\right] \\
& +\frac{A_{\max }}{2}\left[\sin \left(N_{e} \alpha_{2}+\phi_{0}\right)-\sin \left(N_{e} \alpha_{1}+\phi_{0}\right)\right]
\end{aligned}
$$

In reference [23], near the resonance region, it is approximately assumed that $\omega \approx \omega_{n}$. The sweep frequency of rotating speed increases or deceleration is carried out from low to high or from high to low. When the rotating speed frequency is close to the frequency of each order of resonance rotating speed, the blade resonance occurs. The resonance frequency of the blade is equal to the product of the resonance rotating speed frequency and the excitation order. Therefore, the starting and stopping range of sweeping frequency of rotating speed depends on the experimental conditions and will be detailed in Section 3. Based on equation (15), the measured vibration displacement difference functions $\Delta y_{10}$ and $\Delta y_{21}$ of the blade tip passing through sensor TIP1 and sensor TIP0, sensor TIP2 and sensor TIP1 are approximated as follows: 

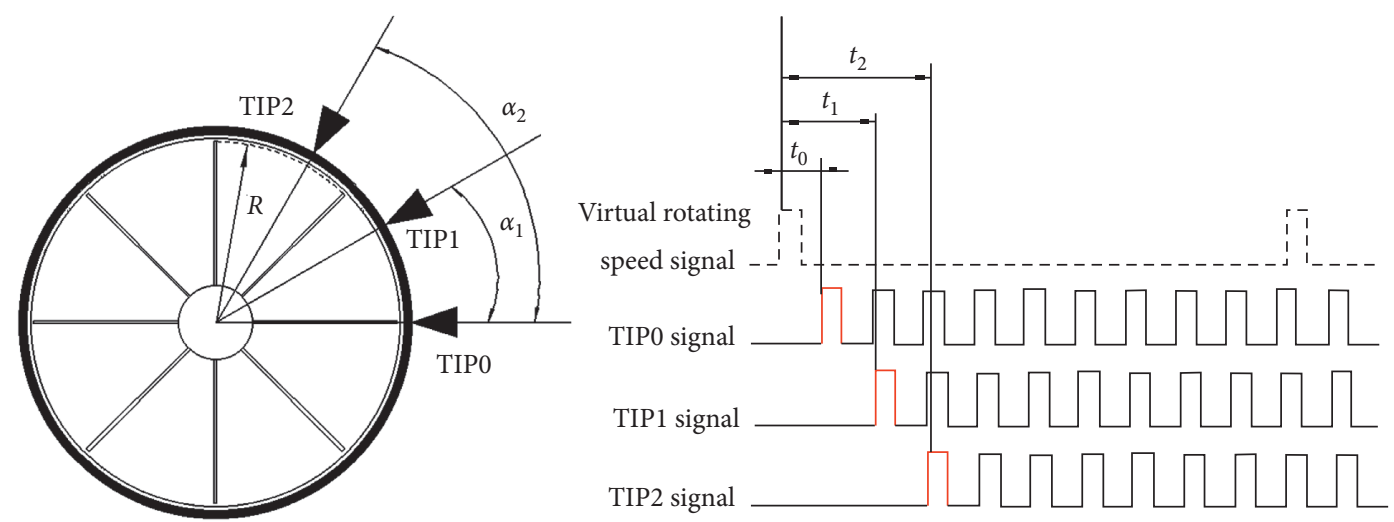

Figure 2: Schematic diagram of sensor layout and blade tip-timing signal of the improved two-parameter method.

$$
\begin{aligned}
\Delta y_{10}= & \frac{\left(y_{1}-y_{0}\right)}{\sin \left(N_{e} \alpha_{1} / 2\right)} \approx A_{\max } \sin \left[\frac{3 \pi}{2}-\left(\frac{4 \phi(\omega)-N_{e} \alpha_{1}-2 \phi_{0}}{2}\right)\right]+\frac{A_{\max }}{2} \frac{\left[\sin \left(N_{e} \alpha_{1}+\phi_{0}\right)-\sin \left(\phi_{0}\right)\right]}{\sin \left(N_{e} \alpha_{1} / 2\right)} \\
\Delta y_{21}= & \frac{\left(y_{2}-y_{1}\right)}{\sin \left(N_{e}\left(\alpha_{2}-\alpha_{1}\right) / 2\right)} \approx A_{\max } \sin \left[\frac{3 \pi}{2}-\left(\frac{4 \phi(\omega)-N_{e}\left(\alpha_{1}+\alpha_{2}\right)-2 \phi_{0}}{2}\right)\right] \\
& +\frac{A_{\max }}{2} \frac{\left[\sin \left(N_{e} \alpha_{2}+\phi_{0}\right)-\sin \left(N_{e} \alpha_{1}+\phi_{0}\right)\right]}{\sin \left(N_{e}\left(\alpha_{2}-\alpha_{1}\right) / 2\right)}
\end{aligned}
$$

Since the approximation is introduced into equation (16), there is an error between the theoretical value and the identification value, which affects the identification accuracy. In the following, an accurate improved two-parameter method is described.

The vibration displacement of the same blade obtained by the three sensors satisfies the following conditions:

$$
\left\{\begin{array}{l}
y_{1}+R \Omega t_{01}-y_{0}=R \alpha_{1} \\
y_{2}+R \Omega t_{12}-y_{1}=R\left(\alpha_{2}-\alpha_{1}\right)
\end{array}\right.
$$

Here, $R$ is the radial distance between the blade tip and the rotation axis, $t_{01}$ is the time the blade tip rotates from TIP0 to TIP1, and $t_{12}$ is the time the blade tip rotates from TIP1 to TIP2. Let $t_{01}^{\prime}$ be the time the blade rotates from TIP0 to TIP1when the blade is not in vibration and $t_{12}^{\prime}$ be the time the blade rotates from TIP1 to TIP2 when the blade is not in vibration. So, there is the following equation:

$$
\left\{\begin{array}{l}
t_{01}^{\prime}=\frac{\alpha}{\Omega}, \\
t_{12}^{\prime}=\frac{\alpha_{2}-\alpha_{1}}{\Omega} .
\end{array}\right.
$$

So, equation (17) becomes

$$
\left\{\begin{array}{l}
y_{1}-y_{0}=R \Omega\left(t_{01}^{\prime}-t_{01}\right) \\
y_{2}-y_{1}=R \Omega\left(t_{12}^{\prime}-t_{12}\right) .
\end{array}\right.
$$

Also,

$$
\frac{\omega}{\omega_{n}}=\frac{N_{e} \Omega}{N_{e} \Omega_{n}}=\frac{\Omega}{\Omega_{n}},
$$

where $\Omega_{n}$ is the resonance center rotating speed frequency and can be obtained by fitting the curve $\Delta y-\Omega$ of equation (19) using the Levenberg-Marquardt method [22]. The optimization parameters, minimum value of objective function, and detailed optimization process of the L-M method are shown in reference [25]. Substituting equations (19) and (20) into equation (15), the corrected vibration displacement differences function $\Delta y_{1}$ and $\Delta y_{2}$ of the blade tip passing through sensor TIP1 and sensor TIP0, sensor TIP2 and sensor TIP1 are as follows:

$$
\begin{aligned}
\Delta y_{1}= & \frac{\left(\Omega / \Omega_{n}\right) R \Omega\left(t_{01}^{\prime}-t_{01}\right)}{\sin \left(N_{e} \alpha_{1} / 2\right)} \\
= & A_{\max } \sin \left[\frac{3 \pi}{2}-\left(\frac{4 \phi(\omega)-N_{e} \alpha_{1}-2 \phi_{0}}{2}\right)\right] \\
& +\frac{A_{\max }}{2} \frac{\left[\sin \left(N_{e} \alpha_{1}+\phi_{0}\right)-\sin \left(\phi_{0}\right)\right]}{\sin \left(N_{e} \alpha_{1} / 2\right)}, \\
\Delta y_{2}= & \frac{\left(\Omega / \Omega_{n}\right) R \Omega\left(t_{12}^{\prime}-t_{12}\right)}{\sin \left(N_{e}\left(\alpha_{2}-\alpha_{1}\right) / 2\right)} \\
= & A_{\max } \sin \left[\frac{3 \pi}{2}-\left(\frac{4 \phi(\omega)-N_{e}\left(\alpha_{1}+\alpha_{2}\right)-2 \phi_{0}}{2}\right)\right] \\
& +\frac{A_{\max }}{2} \frac{\left[\sin \left(N_{e} \alpha_{2}+\phi_{0}\right)-\sin \left(N_{e} \alpha_{1}+\phi_{0}\right)\right]}{\sin \left(N_{e}\left(\alpha_{2}-\alpha_{1}\right) / 2\right)} .
\end{aligned}
$$


Equation (21) is a completely elliptic equation. According to the ellipse transformation, in the $x-y$ coordinate system, the long and short axes of the standard ellipses are $a$ and $b$, respectively, $t$ is the parameter of the ellipse, and the standard equation or parametric equation of the ellipse is shown as follows:

$$
\begin{aligned}
& \frac{x^{2}}{a^{2}}+\frac{y^{2}}{b^{2}}=1, \\
& \left\{\begin{array}{l}
x=a \cdot \cos t, \\
y=b \cdot \cos t .
\end{array}\right.
\end{aligned}
$$

The standard ellipse is rotated counterclockwise by $\theta$ degree around the center, and then the center is shifted to the coordinate $\left(x_{0}, y_{0}\right)$ to obtain the elliptic equation in the $x^{\prime}-$ $y^{\prime}$ coordinate system as shown in the following equation:

$$
\left\{\begin{array}{l}
x^{\prime}=x \cos \theta+y \sin \theta+x_{0}, \\
y^{\prime}=y \cdot \cos \theta-x \cdot \sin \theta+y_{0} .
\end{array}\right.
$$

If $\theta=-45^{\circ}$, according to the auxiliary angle formula, equation (23) is simplified as follows:

$$
\left\{\begin{array}{l}
x^{\prime}=\sqrt{\frac{a^{2}+b^{2}}{2}} \sin (t-\gamma)+x_{0}, \\
y^{\prime}=\sqrt{\frac{a^{2}+b^{2}}{2}} \sin (t+\gamma)+y_{0} .
\end{array}\right.
$$

Here, $\gamma$ is the auxiliary angle and $\tan \gamma=a / b$. If $\theta=45^{\circ}$, the obtained equation is similar to equation (24). Comparing equations (21) and (24), an ellipse having a major axis inclination of $-45^{\circ}$ is drawn with equation (21) as the $x$ axis and the $y$ axis, respectively. The two signals at left end of the equal sign in equation (21), respectively, correspond to $x^{\prime}(i)$ and $y^{\prime}(i)$ where $i=1, \ldots, n$ ( $n$ represents the number of signal data points). The parameters $a, b, x_{0}$, and $y_{0}$ of the ellipse are obtained by least square fitting.

The synchronous vibration amplitude $A_{\max }$ and excitation order $N_{e}$ of the blade are obtained as follows:

$$
\begin{gathered}
A_{\max }=\sqrt{\frac{a^{2}+b^{2}}{2}}, \\
N_{e}=\frac{2 \gamma}{\left(\alpha_{2} / 2\right)} .
\end{gathered}
$$

Here, the phase difference $2 \gamma$ is determined by equation (27). Because $N_{e} \leq 2 \times 360^{\circ} / \alpha_{2}$, the sensor installation angle $\alpha_{2}$ should not be too large.

$$
2 \gamma= \begin{cases}\arcsin \left(\frac{2 \mathrm{ab}}{a^{2}+b^{2}}\right), & \text { first quadrant, } \\ 180-\arcsin \left(\frac{2 \mathrm{ab}}{a^{2}+b^{2}}\right), & \text { second quadrant, } \\ 180+\arcsin \left(\frac{2 \mathrm{ab}}{a^{2}+b^{2}}\right), & \text { third quadrant, } \\ 360-\arcsin \left(\frac{2 \mathrm{ab}}{a^{2}+b^{2}}\right), & \text { fourth quadrant. }\end{cases}
$$

According to the resonance rotating speed $\Omega_{n}$ and the excitation order $N_{e}$, the resonance angular frequency $\omega_{n}=$ $N_{e} \Omega_{n}$ of the blade can be obtained. The initial phase $\phi_{0}$ can be solved by the following equation:

\section{Identification Accuracy Verification and Comparative Analysis}

The first-order bending vibration of a blade can be equivalent to a single degree of freedom spring-damping vibration model. It is assumed that the blade is a simple harmonic vibration, the resonance frequency is $143.244 \mathrm{~Hz}$, the vibration amplitude is $1 \mathrm{~mm}$, the excitation order is 12 , the initial phase is $-7.2^{\circ}$, and the constant offset is $0.2 \mathrm{~mm}$. In order to keep the resonance frequency within the sweep frequency range, the sweep rotating speed frequency range is from $10 \mathrm{~Hz}$ to $14 \mathrm{~Hz}$.
3.1. Simulation Condition without Random Error. The blade vibration parameters are identified by the traditional twoparameter method. The installation angle of the once-per revolution sensor TIP is $0^{\circ}$. The installation angle between the blade tip-timing sensor TIP0 and the once-per revolution sensor TIP of the traditional two-parameter method is $0^{\circ}$, and the installation angle between the tip-timing sensor TIP1 and TIP0 is $20^{\circ}$. The blade vibration displacement curve measured by two BTT sensors with the traditional twoparameter method is shown in Figure 3. Based on the blade vibration displacement curve, the resonance rotating speed frequency (RRSF) of the blade calculated by the method in 


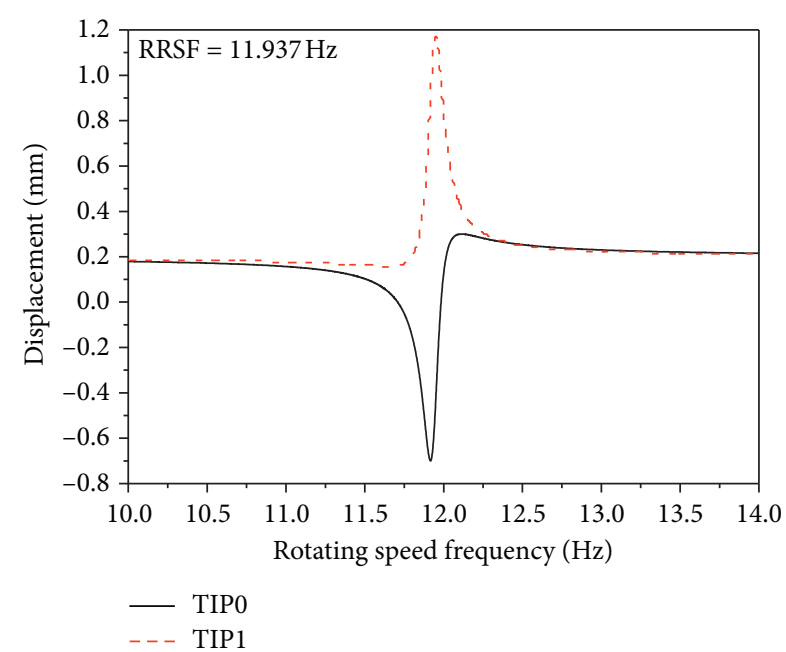

FIgURE 3: Blade vibration displacement curve by two BTT sensors.

reference [14] is $11.937 \mathrm{~Hz}$. The displacements measured by the two sensors are taken as abscissa and ordinate, respectively, and the least square method is used for ellipse fitting. The measurement curve and fitting curve are shown in Figure 4. It can be seen from Figure 4 that there is a certain error between the fitting curve and the measurement curve. The vibration amplitude is $1.0000247 \mathrm{~mm}$, the excitation order is 12.00000292 , the initial phase is $-6.925^{\circ}$, the constant offset is $0.199949 \mathrm{~mm}$, and the resonance frequency is 143.244 Hz, which are obtained by identifying the vibration parameters. The identification value of vibration parameters is close to the theoretical value.

The blade vibration parameters are identified by the improved two-parameter method in reference [23] and the new improved two-parameter method. The installation angle of the BTT sensor TIP0 is $0^{\circ}$. The angle between the sensor TIP0 and sensor TIP1, the sensor TIP0 and sensor TIP2 of the two improved two-parameter methods is $20^{\circ}$ and $40^{\circ}$. Because the vibration displacement difference curve can not form an accurate ellipse, therefore, the method proposed in this paper is used to correct the difference curve. The ratio of the rotating speed frequency to the resonance rotating speed frequency $\Omega / \Omega_{n}$ is used to correct the measured data in equation (21). The corrected vibration displacement difference curve can form an accurate ellipse. The measured data, corrected data, and relative error of vibration displacement difference between sensor TIP1 and sensor TIP0, sensor TIP2 and sensor TIP1 are shown in Figures 5 and 6, respectively. It can be seen from Figures 5 and 6 that the trend of measured curve and corrected curve is consistent. Through the local enlarged drawing and relative error curve, it can be found that the closer to the resonance region, the smaller the relative error of the vibration displacement difference and the farther away from the resonance region, the larger the relative error of the vibration displacement difference. The two groups of measured displacement difference data and corrected displacement difference data are used as abscissa and ordinate, respectively, and the least square method is used for ellipse fitting. Ellipse fitting results

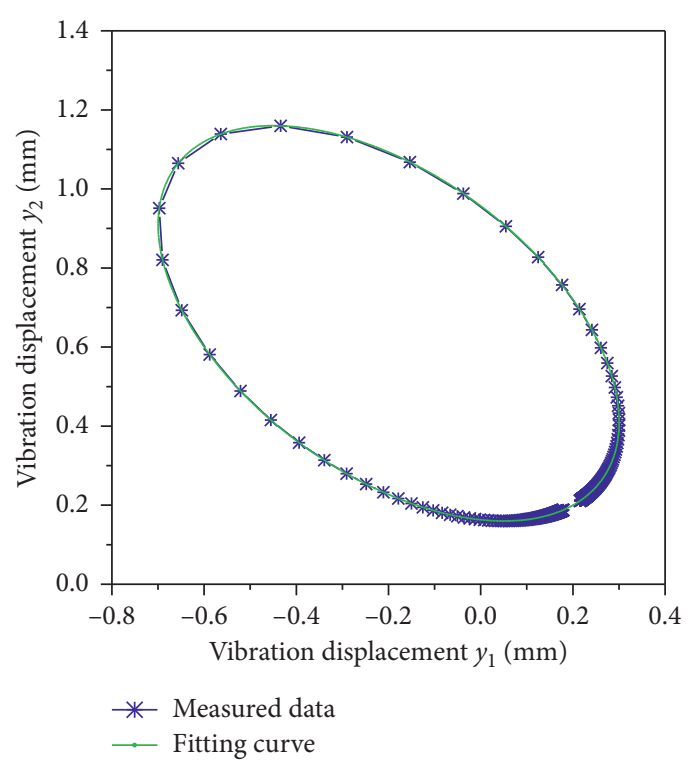

FIgURE 4: Measurement curve and fitting curve.

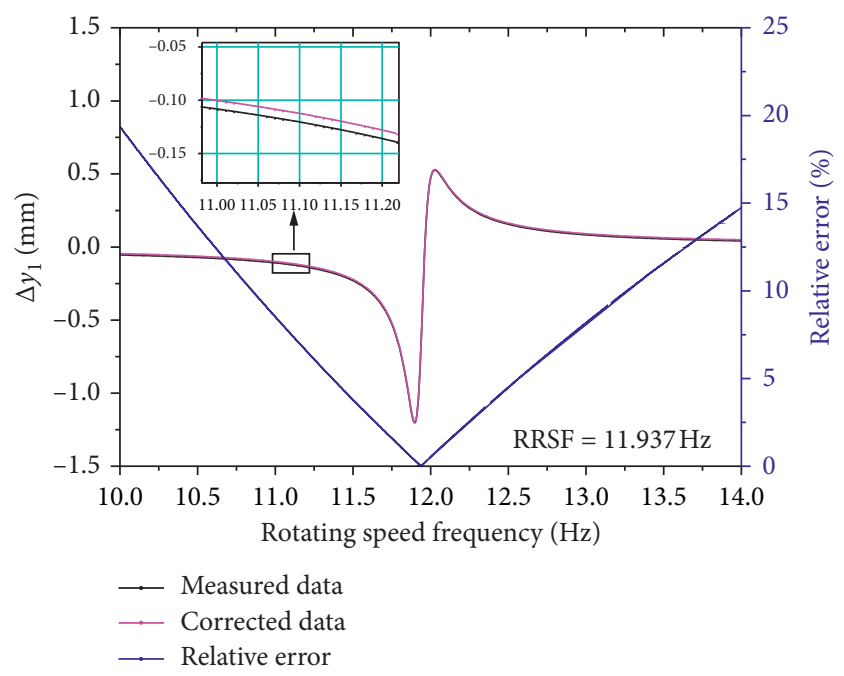

FIgURE 5: Measured data, corrected data, and relative error of $\Delta y_{1}$.

are shown in Figure 7. By observing the local zoom of Figure 7, it can be found that there are some errors between the approximate fitting elliptic curve using the method in reference [23] and the corrected fitting elliptic curve using the method in this paper.

Due to the existence of errors, there are some differences in the results of vibration parameter identification. The theoretical value of vibration parameters and identification value of vibration parameters by three methods are shown in Table 1. From the identification results in Table 1, it is found that the identification accuracy of the new improved twoparameter method is higher than that of the traditional twoparameter method without introducing errors, while the identification accuracy of the traditional two-parameter method is also higher than that of the improved two-parameter method in reference [23]. The identification value of 


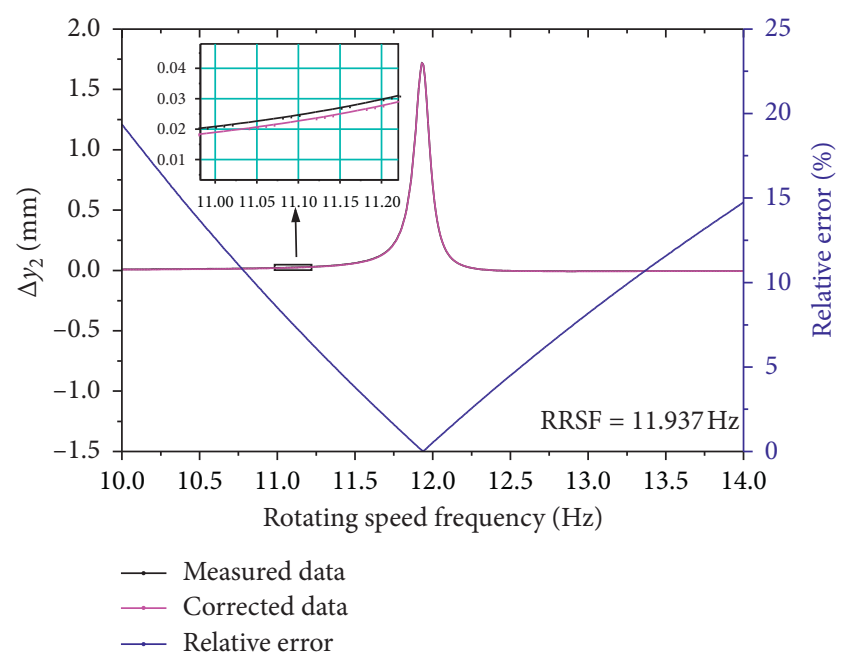

FIgURE 6: Measured data, corrected data, and relative error of $\Delta y_{2}$.

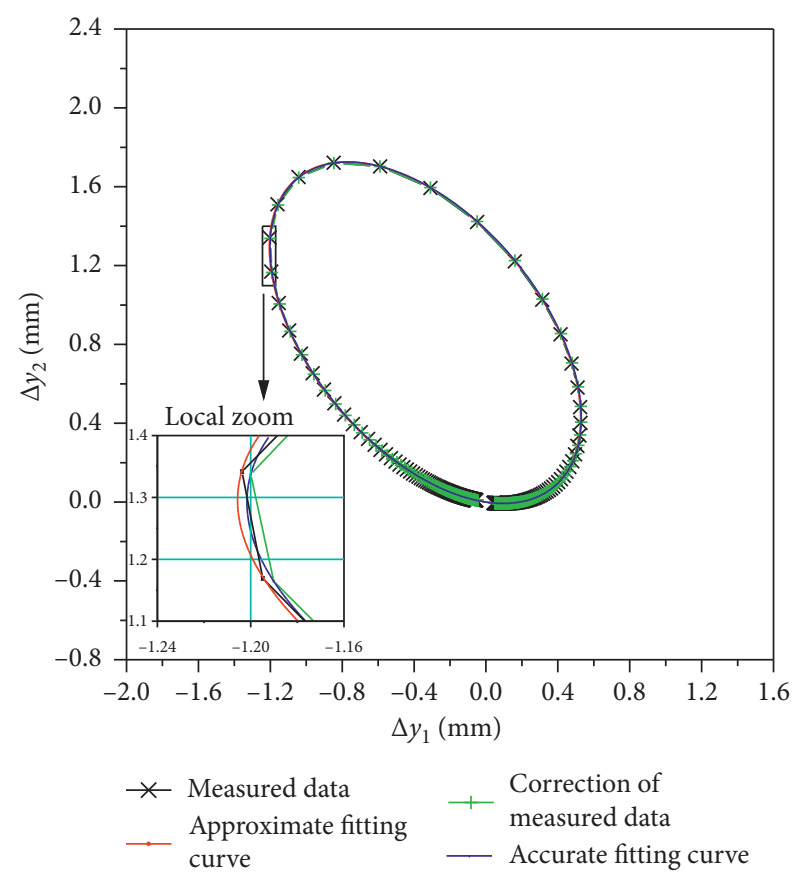

Figure 7: Approximate and accurate fitting curve.

the new improved two-parameter method proposed in this paper is consistent with the theoretical value. The ellipse approximation of the traditional two-parameter method and the improved two-parameter method in reference [23] has little influence on the identification accuracy of vibration amplitude and excitation order but has some influence on the identification accuracy of the initial phase. The advantages of the method in this paper and the method in reference [23] are that the once-per revolution sensor is not necessary and the disadvantages are that the constant deviation can not be identified.

3.2. Simulation Condition with Random Error. In the following, a random error of white gaussian noise with a relative error of $0-15 \%$ is introduced into the simulated measurement vibration signal of each sensor, respectively. The method proposed in this paper and the method in reference [23] are, respectively, used to identify the vibration parameters of the blade, and the identification accuracy of the two methods is compared under the condition of the sensor measurement error. The influence of sensor measurement error on the identification accuracy of vibration parameters of the two methods is analyzed. The relative error of vibration parameters identification accuracy of the two methods is shown in Figure 8. It can be seen from Figure 8 that when no error $(0 \%)$ is introduced into the simulation measurement data, there is a certain theoretical error in the initial phase of vibration identified by the method in reference [23]. The sensor measurement error has the greatest impact on the identification accuracy of the initial phase of the vibration, followed by the vibration amplitude, and has little impact on the identification accuracy of the excitation order. With the increase in the random error of sensor measurement, the relative error of identification of the initial phase of vibration of the two methods increases and is accompanied by oscillation, the relative error of the vibration amplitude identification is stable within $6 \%$, and the relative error of the excitation order identification is stable within $0.7 \%$. The identification accuracy of vibration amplitude and excitation order of the two methods are basically the same. However, the identification accuracy of the initial phase of vibration of this method is obviously higher than that of the method in reference [23].

\section{Vibration Parameters Identification Based on New Improved Two-Parameter Method}

4.1. Vibration Test Principle of Rotating Bladed Disk System Based on Blade Tip-Timing Technology. In the previous section, the identification accuracy of the new improved two-parameter method proposed in this paper is verified by numerical simulation. In this section, the feasibility of this method is verified by vibration testing. The blade tip-timing vibration measurement system is shown in Figure 9 and is mainly composed of five devices: bladed disk rotor system, sensors, excitation system, signal acquisition box, and computer data processing software. The basic principle of the vibration measurement based on blade tip-timing is that the servo motor drives the straight blades disk to rotate through the belt drive, the number of blades is 8 , and the radius of blades tip is $217 \mathrm{~mm}$. The electromagnetic exciter is used to apply the exciting force to the blade passing through it in the test device. The number of exciters is 2 and which are arranged uniformly along the circumference to excite the blade vibration, and the blade vibration order is an integral multiple of 2. The OPR sensor TIP is mounted in the horizontal direction on the opposite of the rotating shaft. The mounting position of the OPR sensor is aligned with the first BTT sensor TIP0. A reflective mark is made at a certain position on the circumference of the rotating shaft. When the laser light emitted by the OPR sensor is irradiated to the reflective mark on the rotating shaft, the rotational speed signal of the bladed disk is detected, thereby obtaining the 
TABLe 1: Parameter identification results of three methods.

\begin{tabular}{|c|c|c|c|c|}
\hline $\begin{array}{l}\text { Vibration } \\
\text { parameters }\end{array}$ & $\begin{array}{c}\text { Theoretical } \\
\text { value }\end{array}$ & $\begin{array}{l}\text { Traditional two-parameter } \\
\text { method }\end{array}$ & $\begin{array}{c}\text { Improved two-parameter method in } \\
\text { reference [23] }\end{array}$ & $\begin{array}{l}\text { New improved two- } \\
\text { parameter method }\end{array}$ \\
\hline $\begin{array}{l}\text { Vibration amplitude } \\
(\mathrm{mm})\end{array}$ & 1 & 1.0000247 & 1.0000250 & 1 \\
\hline Excitation order & 12 & 12.00000292 & 12.00001197 & 12 \\
\hline Initial phase $\left(^{\circ}\right)$ & -7.2 & -6.925 & -6.915 & -7.2 \\
\hline $\begin{array}{l}\text { Resonance frequency } \\
(\mathrm{Hz})\end{array}$ & 143.244 & 143.244 & 143.244 & 143.244 \\
\hline $\begin{array}{l}\text { Constant deviation } \\
(\mathrm{mm})\end{array}$ & 0.2 & 0.199949 & Unrecognized & Unrecognized \\
\hline
\end{tabular}

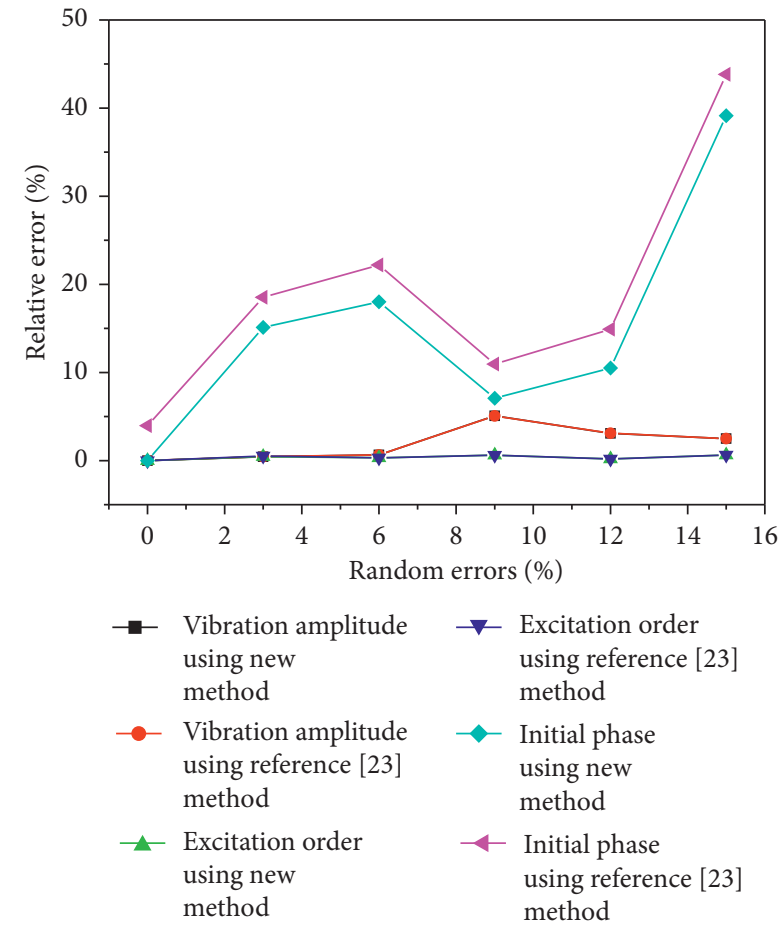

FIGURE 8: Relative error of vibration parameters identification by two methods.

rotational frequency of the bladed disk. The purpose of the OPR sensor installed on the test device is to verify the accuracy of the blade tip-timing method without OPR sensor proposed in this paper. Three eddy current sensors TIP0, TIP1, and TIP 2 are installed in turn at $20^{\circ}$ intervals on the sensors bracket. The voltage-time pulse signals of the rotating blades tip when they reach each eddy current sensor are collected by the signal acquisition system. These signals are fed back to the data acquisition and processing software of the computer.

\subsection{Synchronous Vibration Parameters Identification of Sweep} Variable Rotating Speed Blade. The sweep variable rotating speed frequency range of the bladed disk is $5-60 \mathrm{~Hz}$; that is, the initial rotation speed of the bladed disk is $300 \mathrm{r} / \mathrm{min}$ and the end rotation speed is $3600 \mathrm{r} / \mathrm{min}$. In the data acquisition and processing software, the voltage-time (blade tip-timing) signals of the blades in the sweep variable rotating speed frequency range measured by the sensors TIP0, TIP1, and TIP2 are collected. Then, the blade tip-timing signal ofNo. 0 blade in the bladed disk is converted into a corresponding vibration displacement difference-rotational number signal. Figure 10 shows the curve of the rotation speed and vibration displacement difference with the rotation number. It can be seen from Figure 10 that there are two obvious resonance regions, and the resonance speed is about $960 \mathrm{r} / \mathrm{min}$ and $2700 \mathrm{r} /$ min, respectively.

Two resonance regions in Figure 10 are selected for research. The central rotational speed frequency of two resonance regions is about $16 \mathrm{~Hz}$ and $45 \mathrm{~Hz}$, respectively. The Levenberg-Marquardt method is used to fit the curves $\Delta y-\Omega$ of equation (19), and the accurate resonance rotational speed frequency is obtained, which is $15.96 \mathrm{~Hz}$ and $44.77 \mathrm{~Hz}$, respectively. The rotational speed frequency range of the two resonance regions is determined as $12-20 \mathrm{~Hz}$ and $42-48 \mathrm{~Hz}$, respectively. The accurate resonance rotational speed frequency is substituted into equation (21), and the corrected change curve of the vibration displacement difference of the two resonance regions with the rotational speed frequency is shown in Figure 11.

The accurate least square ellipse fitting is used to fit the vibration displacement difference data of two resonance regions, and the fitting results are shown in Figure 12. When the curve is drawn with the value of $\Delta y_{1}$ as the abscissa and $\Delta y_{2}$ as the ordinate, the amplitudes of $\Delta y_{1}$ and $\Delta y_{2}$ in the nonresonant region are relatively small and their changes are relatively small and close to 0 , so the data points in the nonresonant region are relatively concentrated. However, the amplitudes of $\Delta y_{1}$ and $\Delta y_{2}$ near the resonance region are relatively large and vary greatly, so the data points in the resonance region are relatively scattered. The identification results of the synchronous vibration parameters of the blades near the two resonance rotational speeds based on equations (25)-(28) are shown in Table 2.

From Table 2, it can be seen that the standard errors of the vibration amplitude, excitation order, resonance speed frequency, resonance frequency, and initial phase identified by the two methods are $0.0033,0.0044,0.0004,0.0010$, and 0.35 , respectively. In the resonance region 1 , the standard errors on the initial phases -7.29 and -7.06 of the two methods are 0.35 , and they are also compatible with each other. However, in resonance region 2, the initial phases of 


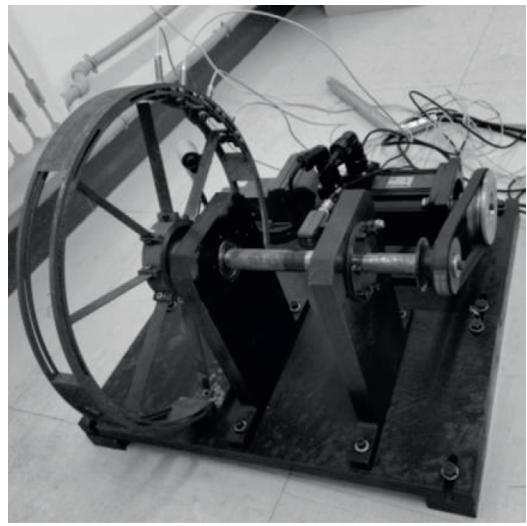

(a)

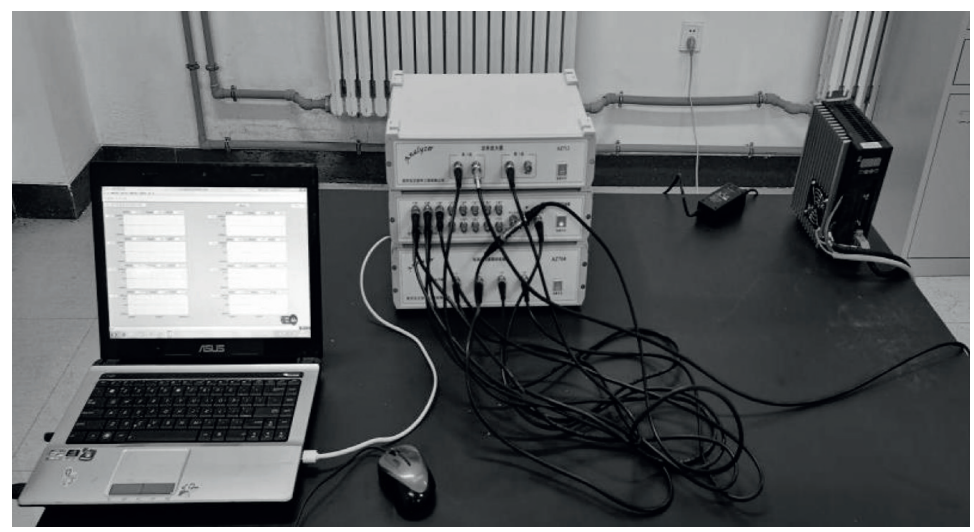

(b)

Figure 9: Blade tip-timing vibration measurement system: (a) vibration test device; (b) signal acquisition and processing system.

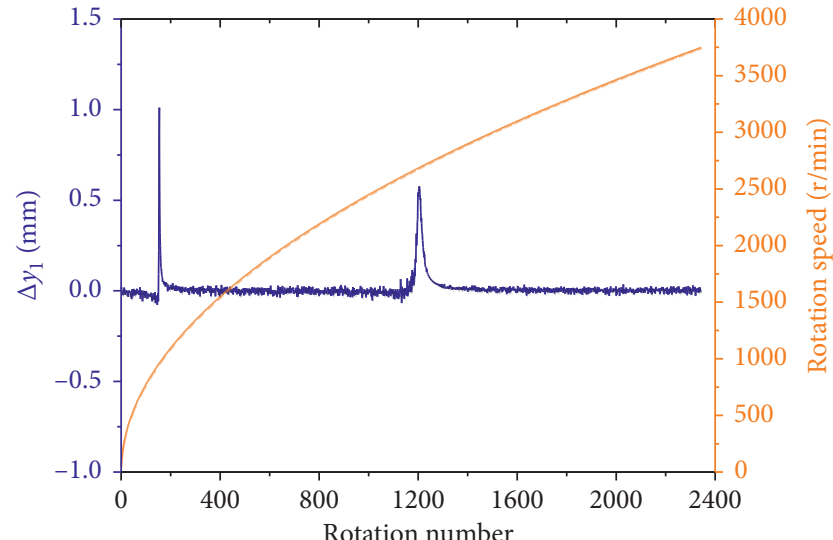

- Vibration displacement - Rotation speed

(a)

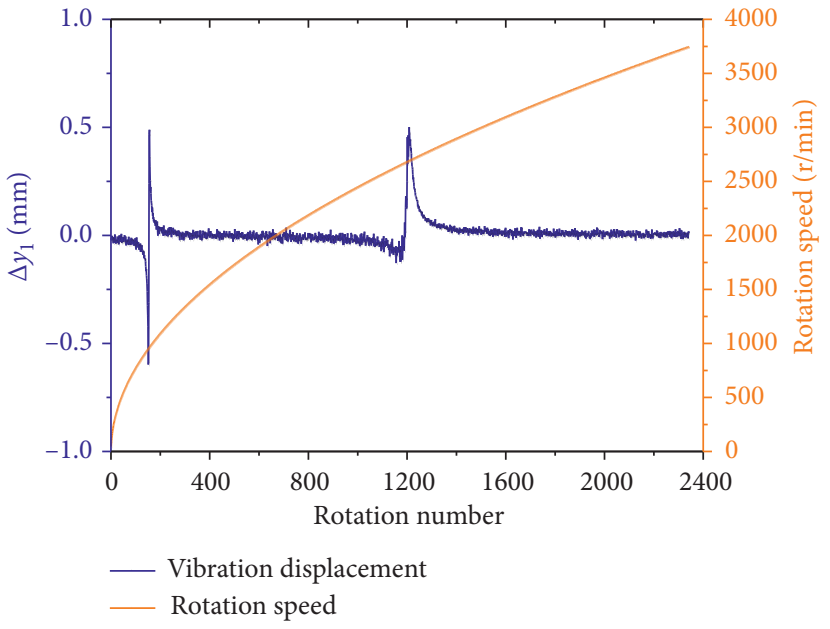

(b)

FIGURE 10: Curve of the rotational speed and vibration displacement difference with rotation number: (a) vibration displacement difference between TIP1 and TIP0; (b) vibration displacement difference between TIP2 and TIP0.

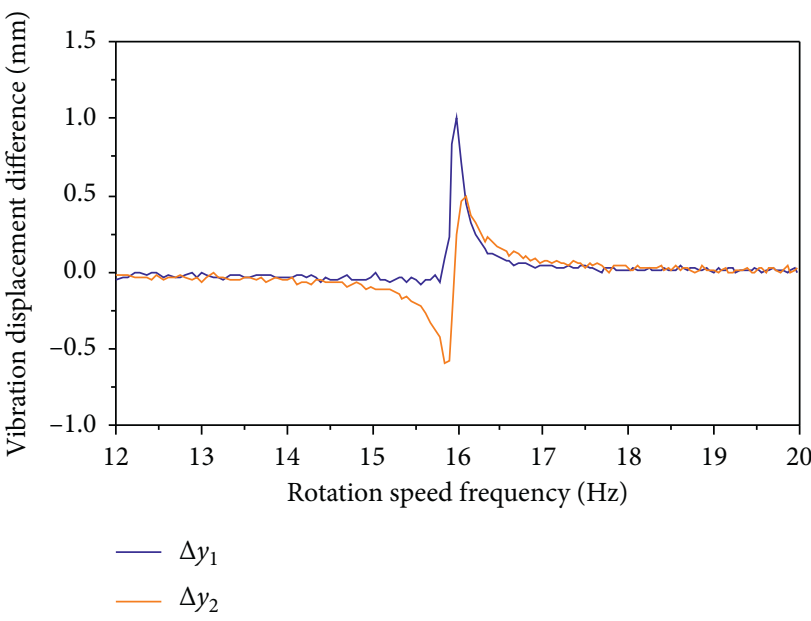

(a)

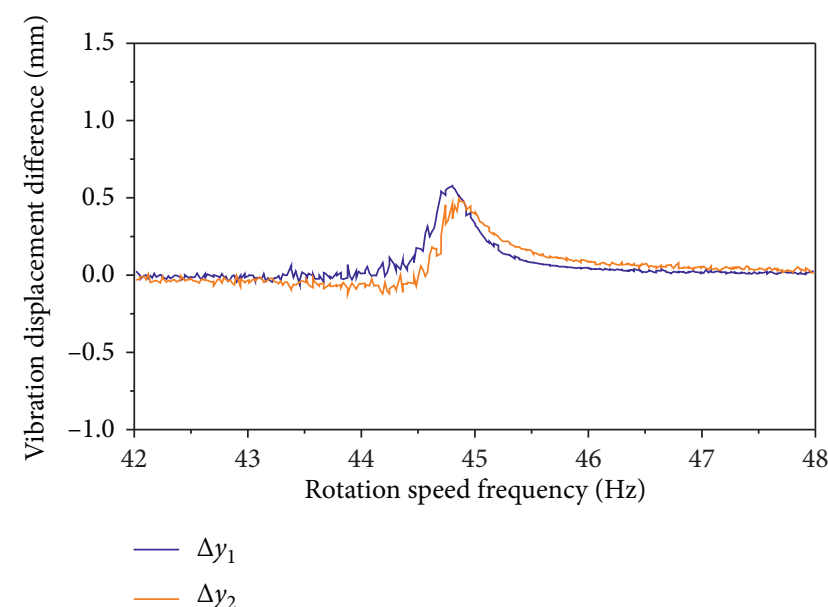

(b)

FIGURE 11: Corrected change curve of the vibration displacement difference of the two resonance regions with the rotation speed frequency: (a) resonance region 1 and (b) resonance region 2 . 


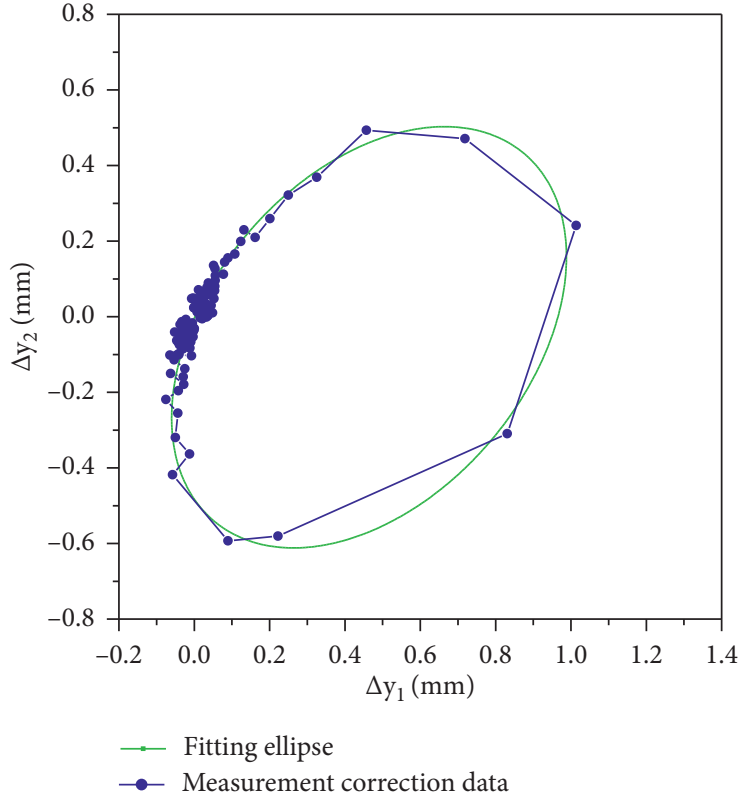

(a)

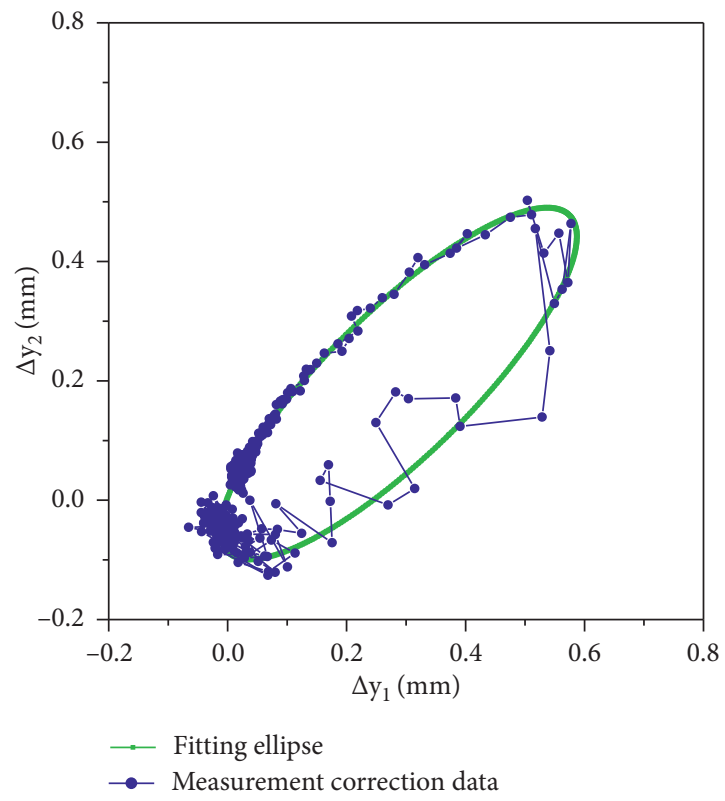

(b)

FIGURE 12: Ellipse fitting results of vibration displacement difference in two resonance regions: (a) resonance region 1; (b) resonance region 2.

TABLE 2: Identification results of synchronous vibration parameter based on test data.

\begin{tabular}{|c|c|c|c|c|c|}
\hline \multirow[b]{2}{*}{ Identification parameters } & \multicolumn{2}{|c|}{ Resonance region 1} & \multicolumn{2}{|c|}{ Resonance region 2} & \multirow{2}{*}{$\begin{array}{l}\text { Standard errors of two } \\
\text { methods }\end{array}$} \\
\hline & $\begin{array}{l}\text { New } \\
\text { method }\end{array}$ & $\begin{array}{l}\text { Reference [23] } \\
\text { method }\end{array}$ & $\begin{array}{l}\text { New } \\
\text { method }\end{array}$ & $\begin{array}{l}\text { Reference [23] } \\
\text { method }\end{array}$ & \\
\hline Vibration amplitude (mm) & 0.9769 & 0.9763 & 1.0360 & 1.0406 & 0.0033 \\
\hline Excitation order & 4.1026 & 4.1039 & 2.0338 & 2.0277 & 0.0044 \\
\hline $\begin{array}{l}\text { Resonance speed frequency } \\
(\mathrm{Hz})\end{array}$ & 15.9606 & 15.9603 & 44.7713 & 44.7709 & 0.0004 \\
\hline Resonance frequency $(\mathrm{Hz})$ & 63.8424 & 63.8412 & 89.5426 & 89.5418 & 0.0010 \\
\hline Initial phase $\left({ }^{\circ}\right)$ & -7.29 & -7.06 & -0.58 & -0.15 & 0.35 \\
\hline
\end{tabular}

-0.58 and -0.15 of the two methods with a standard error of 0.35 could actually be different. The vibration parameter identification results of the new method in this paper are in good agreement with the method in reference [23], except for the initial phase of resonance region 2 . The identification result is consistent with the previous simulation identification result, which further illustrates that the initial phase identified by the method of reference [23] has a large error. According to the Campbell diagram of blade No. 0, the resonance rotation speed frequency of blade 4-order vibration is about $16 \mathrm{~Hz}$ and the resonance rotation speed frequency of the 2-order vibration is about $45 \mathrm{~Hz}$, which further verifies the feasibility and accuracy of the new method proposed in this paper.

\section{Conclusions}

In this article, a new improved two-parameter method for synchronous vibration parameters identification of sweep variable rotating speed blades based on blade tip-timing is proposed. Compared with the traditional two-parameter method, the method requires no OPR sensor which makes it more generally applicable in the condition where the OPR sensor is difficult to install. Three eddy current sensors are required to identify the excitation order, vibration amplitude, the resonance speed frequency, the resonance frequency, and the initial phase of the blades. Numerical simulation analysis of blade synchronous vibration parameters identification are presented, and the identification error of the method is investigated. Experiments are carried out based on the blade tip-timing rotor test device. The simulation results indicate that the identification accuracy of this method is higher than that of the traditional two-parameter method and the improved method in reference, especially in the identification of the vibration initial phase. The experimental results show that the synchronous vibration parameter identification of sweep variable rotating speed blade is achieved based on the new improved twoparameter method which requires no OPR sensor, and the identification results are consistent with the results of the 
improved two-parameter method in reference [23], except for the initial phase. The new improved two-parameter method proposed in this paper provides a theoretical basis for the practical application of vibration parameters identification technology of blade tip-timing without the OPR sensor.

\section{Data Availability}

The data used to support the findings of this paper are available from the corresponding author upon request.

\section{Conflicts of Interest}

The authors declare that there are no conflicts of interest regarding the publication of this paper.

\section{Acknowledgments}

This article was supported by National Natural Science Foundation of China (no. 51505206), Liaoning Provincial Natural Science Foundation Guidance Program Project (no. 2019-ZD-0694), Liaoning Provincial Department of Education Scientific Research General Project (no. L2014246) and Liaoning University of Technology Teacher Research Start Fund Project (no. X201202). The authors are grateful to them.

\section{References}

[1] L. Han, C. Chen, T. Y. Guo et al., "Probability-based service safety life prediction approach of raw and treated turbine blades regarding combined cycle fatigue," Aerospace Science and Technology, vol. 110, no. 7, Article ID 106513, 2021.

[2] C. Lu, C. W. Fei, H. Li, and H. T. Liu, "Moving extremum surrogate modeling strategy for dynamic reliability estimation of turbine blisk with multi-physics fields," Aerospace Science and Technology, vol. 106, Article ID 106112, 2020.

[3] L. M. Amoo, "On the design and structural analysis of jet engine fan blade structures," Progress in Aerospace Sciences, vol. 60, pp. 1-11, 2013.

[4] S. Heath, S. Mansfield, and P. Loftus, "Turbo machinery blade tip-timing measurement techniques 90th symp," AGARDPEP on Advanced Non-Intrusive Instrumentation for Propulsion Engines Brussels, vol. 32, pp. 1-9, 1997.

[5] K. R. Grant and P. Ivey, "The experimental validation of tiptiming techniques for condition monitoring of HCF in gas turbine aero-engines," in Proceedings of the 38th AIAA/ ASME/SAE/ASEE Joint Propulsion Conference and Exhibit, Indianapolis, IA, USA, July 2002.

[6] J. Lin, Z. Hu, Z. S. Chen, and Y. Yang, "Sparse reconstruction of blade tip-timing signals for multi-mode blade vibration monitoring," Mechanical Systems and Signal Processing, vol. 81, pp. 250-258, 2016.

[7] G. Battiato, C. M. Firrone, and T. M. Berruti, "A benchmark for tip timing measurement of forced response in rotating bladed disks," in Proceedings of the International Conference on Engineering Vibration, 2015.

[8] L. Yue, H. Liu, C. Zang, D. Wang, W. Hu, and L. Wang, "The parameter identification method of blade asynchronous vibration under sweep speed excitation," Journal of Physics: Conference Series, vol. 744, Article ID 012051, 2016.

[9] P. Wang, D. Karg, Z. Fan, R. X. Gao, K. Kwolek, and A. Consiglio, "Non-contact identification of rotating blade vibration," Mechanical Engineering Journal, vol. 2, no. 3, pp. 15-00025, 2015.

[10] O. Freund, M. Montgomery, M. Mittelbach, and J. R. Seume, "Non-contact test set-up for aeroelasticity in a rotating turbomachine combining a novel acoustic excitation system with tip-timing," Measurement Science and Technology, vol. 25, no. 3, Article ID 035008, 2014.

[11] F. Wu, L. Liang, J. Xing, L. Wang, and L. Jia, "Blade tip timing vibration monitoring method based on fiber Bragg grating," Photonic Sensors, vol. 4, no. 2, pp. 188-192, 2014.

[12] G. Battiato, C. M. Firrone, and T. M. Berruti, "Forced response of rotating bladed disks: blade tip-timing measurements," Mechanical Systems and Signal Processing, vol. 85, pp. 912-926, 2017.

[13] D. D. Maio and D. J. Ewins, "Experimental measurements of out-of-plane vibrations of a simple blisk design using blade tip timing and scanning LDV measurement methods," $M e$ chanical Systems and Signal Processing, vol. 28, pp. 517-527, 2012.

[14] G. Rigosi, G. Battiato, and T. M. Berruti, "Synchronous vibration parameters identification by tip timing measurements," Mechanics Research Communications, vol. 79, pp. 7-14, 2017.

[15] C. Lawson and P. Ivey, "Tubomachinery blade vibration amplitude measurement through tip timing with capacitance tip clearance probes," Sensors and Actuators A: Physical, vol. 118, no. 1, pp. 14-24, 2005.

[16] S. Heath, "A new technique for identifying synchronous resonances using tip-timing," Journal of Engineering for Gas Turbines \& Power, vol. 122, no. 2, pp. 219-225, 2000.

[17] F. Duan, T. Ouyang, and M. Li, "Research on detecting technology of rotating blade vibration performance parameters," in Proceedings of the 2009 International Conference on Measuring Technology and Mechatronics Automation, Zhangjiajie, Hunan, China, May, 2009.

[18] C. Liu and D. Jiang, "Improved blade tip timing in blade vibration monitoring with torsional vibration of the rotor," Journal of Physics: Conference Series, vol. 364, Article ID 012136, 2012.

[19] G. Ferraris, V. Maisonneuve, and M. Lalanne, "Prediction of the dynamic behavior of non-symmetrical coaxial co- or counter-rotating rotors," Journal of Sound and Vibration, vol. 195, no. 4, pp. 649-666, 1996.

[20] P. Russhard, "Derived once per rev signal generation for blade tip timing systems," in Proceedings of the International Instrumentation Symposium IET, London, UK, June 2014.

[21] K. Chen, W. M. Wang, X. L. Zhang, and Y. Zhang, "New step to improve the accuracy of blade tip timing method without once per revolution," Mechanical Systems and Signal Processing, vol. 134, pp. 1-19, 2019.

[22] H. Guo, F. Duan, and J. Zhang, "Blade resonance parameter identification based on tip-timing method without the onceper revolution sensor," Mechanical Systems and Signal Processing, vol. 66-67, pp. 625-639, 2016.

[23] C. Y. Zhou, H. F. Hu, Y. M. Yang, F. J. Guan, and H. L. Xu, "Research on blade synchronous vibration parameter identification using blade tip timing without the once per revolution sensor," Journal of Mechanical Engineering, vol. 55, no. 13, pp. 64-71, 2019. 
[24] T. Ouyang, F. J. Duan, M. Yan, M. L. Li, Z. C. Zhong, and X. J. Shi, "Research on identifying synchronous blade vibration using two-parameter-plot method," Transducer and Mirosystem Technologies, vol. 29, no. 3, pp. 42-45, 2010.

[25] T. Ouyang, Rotating Blade Vibration Detection and Parameters Identification Technique Using Blade Tip-Timing, Ph.D. thesis, Ph.D. thesis of Tianjin University, Tianjin, China, 2011. 